Review

\title{
Optimal Rule-Based Power Management for Online, Real-Time Applications in HEVs with Multiple Sources and Objectives: A Review
}

\author{
Bedatri Moulik * and Dirk Söffker \\ Chair of Dynamics and Control, University of Duisburg-Essen, Lotharstr. 1, Duisburg 47057, Germany; \\ E-Mail: soeffker@uni-due.de \\ * Author to whom correspondence should be addressed; E-Mail: bedatri.moulik@uni-due.de; \\ Tel.: +49-203-379-3422; Fax: +49-203-379-3027.
}

Academic Editor: Enrico Sciubba

Received: 25 June 2015 / Accepted: 17 August 2015 / Published: 26 August 2015

\begin{abstract}
The field of hybrid vehicles has undergone intensive research and development, primarily due to the increasing concern of depleting resources and increasing pollution. In order to investigate further options to optimize the performance of hybrid vehicles with regards to different criteria, such as fuel economy, battery aging, etc., a detailed state-of-the-art review is presented in this contribution. Different power management and optimization techniques are discussed focusing on rule-based power management and multi-objective optimization techniques. The extent of rule-based power management and optimization in solving battery aging issues is investigated along with an implementation in real-time driving scenarios where no pre-defined drive cycle is followed. The goal of this paper is to illustrate the significance and applications of rule-based power management optimization based on previous contributions.
\end{abstract}

Keywords: rule-based power management; optimization; hybrid electric vehicles

\section{Introduction}

For the past several years, hybrid electric vehicles (HEVs) have mostly implied two power sources with power management approaches responsible for distributing power between these two sources. However, the presence of multiple power sources with a suitable power distribution between components 
makes further reduction in fuel consumption possible. In hybrid powertrains, two different sources of power are considered. As an example, in [1], the drivetrain output mechanically driven by an internal combustion engine (ICE) is supplemented by an electric motor. However, due to the growing trend of all-electric powertrains [2], the efficiency assessment of such powertrains becomes important. According to [1], the efficiency of all-electric hybrid vehicles depends on the capability of the energy storage systems (ESSs), where batteries and supercapacitors are considered as the most common options for vehicular ESSs. As stated in [1], a hybrid drive composed of batteries, supercapacitors and fuel cells could be considered as an appropriate option for advanced hybrid vehicular drives. According to [3], Li-ion batteries possess high energy density, but have relatively poor power density, whereas supercapacitors possess high power density, but lower energy density, along with high charging/discharging efficiency compared to batteries. As a result, hybridization of Li-ion batteries and supercapacitors is considered in [3] to lead to a high performance storage unit. According to [3], the desired operation is when a nearly constant load current is supplied by the battery. This reduces its $I^{2} R$ losses and prevents terminal voltage drops. The dynamic current with zero average is supplied by the supercapacitor in order to match the battery to the load. A fuel cell-battery-supercapacitor combination was also chosen in [4], stating the advantage of this combination. The advantage of the battery-supercapacitor combination has also been stated in [5], where the constraint for the control strategy was to remain charge depleting. Due to the battery-supercapacitor combination, the supercapacitor can take over more dynamic power fluctuations, while the battery takes a low pass filtered profile. According to [5], this can extend the battery life. The advantage of fuel the cell-battery-supercapacitor combination has also been discussed in [6], where fuzzy logic control was used to design energy management strategy for the hybrid powertrain. Along with primary energy sources (such as fuel cells) and ESSs, DC/DC converters are also integral parts of HEVs. The current flow into the DC bus can be controlled with the help of DC/DC converters. The power split ratio between the battery and the fuel cell is obtained by sending the fuel cell net current required to the DC/DC converter. In [7], a supervisory controller is considered along with a combined power management and design optimization for a fuel cell hybrid vehicle. This controller sends the current request to DC/DC converters. Here, the aim was to minimize the fuel consumption by maintaining the battery State of Charge (SoC). by sending the appropriate current request command to the DC/DC converter. The DC/DC converter, which plays a central role in power management, has also been considered in [8] with fuel cell hybrids. However, an integration of three DC/DC converters for all three sources in a fuel cell-battery-supercapacitor powertrain, with at least two bi-directional DC/DC converters, provides more degrees of freedom, as detailed in [3,9]. Power management with DC/DC converter control can be realized with real hardware components, of which emulator hardware [10] has been considered as a less expensive and more compact substitute for complex real hardware. The emulator test-rig developed and implemented in [11] is capable of accurately replicating the vehicle dynamics and can also be used for validating power flow algorithms. This was extended and modified to emulate an entire hybrid powertrain in [12].

An important criteria in HEV design is to achieve an efficient conversion of energy on the powertrain [2], where the effectiveness of the results can be checked with standard drive cycles. In order to achieve this purpose, in [2], the need to design a suitable controller and control strategies 
is emphasized. Therefore, the control strategies can satisfy a number of goals, such as maximum fuel economy, good driving performance, etc. Amongst these power management control strategies, the effectiveness of rule-based power management in real-time supervisory control has been stated in [13]. According to [13], under rule-based power management, deterministic rule-based power management, as an easy to realize method, relies on heuristics to design rules that are generally implemented via look-up tables. An adaptive rule-based power management for optimization of both energy use and emissions is also mentioned. In the case of hybrid vehicles, which involve multi-variables and/or multi-objectives, rules that cover all important trade-offs among different optimization objectives are difficult to design, and therefore, in [14], dynamic programming (DP) is used to understand the deficiency of rules and to serve as a reference to construct improved rules in terms of a fuel economy-emissions optimization problem. In [15], a two-mode, low and high level controller design is considered, which is subjected to an offline control variables' optimization in terms of fuel consumption minimization. Similarly, in [16], a rule-based energy management controller was developed that includes off-line calculation relative to the optimization problem. State-of-charge was the key variable that determined the selection between modes. Some of the earliest works in rule-based control include [17], where optimal energy management deals with power split in series hybrid electric vehicles. A sub-categorization of rule-based control is considered in [2].

Apart from online rule-based power management techniques, various offline optimization algorithms are also available, such as DP [18-20] and GA [21,22]. These methods are capable of producing globally-optimal solutions, and some more sophisticated ones, such as the Non-dominated Sorting Genetic Algorithm II (NSGA II) [23], are also capable of tackling multiple and conflicting objectives, such as the problem of fuel consumption and emissions minimization in [24], fuel consumption and component sizing in [25] and fuel cost and battery health degradation minimization in [26]. The other category of power management optimization techniques includes those that can be implemented in real-time, such as [27], where along with a real-time controller, two optimization goals of fuel consumption and battery SoC deviation minimization were considered. Model predictive control (MPC) has also been widely used for real-time control. For instance, in [28], a non-linear MPC was considered to carry out the task of fuel efficiency maximization with battery SoC, vehicle relative position and performance as constraints. Equivalent consumption minimization strategies (ECMS) [29,30], on the other hand, are instantaneous power management strategies that can be implemented online, offline, as well as in real time. A detailed classification of power management optimization methods has been given in [31]. An interesting procedure to evaluate the performance of online power management strategies in terms of fuel consumption and computational cost was carried out in [32]. Here, nine participating teams were provided with a hybrid vehicle model. Each of the power management solutions presented by participants were tested for two realistic driving cycles not known to the participants. The comparison results were obtained for rule-based $v s$. ECMS. The best solution obtained was compared with global, offline optimization.

The non-optimality resulting from conventional rule-based power management with respect to off-line techniques [31] leads to the consideration of improvement methods in [33]. Here, a parameter optimization using genetic algorithms (GA) was considered to determine the optimal control variables for fixed parameters. Using this as the baseline, an equivalent consumption management strategy (ECMS) 
to dynamically control the controller parameters and a route-based strategy to dynamically control the parameters for current and predicted future routes were developed. The combination of rule-based control strategies with optimal power management strategies has also been considered in [34]. Here, the performance of the rule-based strategy was assessed by comparing with GA, and the suitability of using GA was established by comparing with dynamic programming (DP). Additionally, power delivered by primary source and SoC deviation are defined as functions of traction power. These functional dependencies were integrated as look-up tables (LUTs) for an online implementation. The values in the LUT were defined by optimizing in terms of fuel efficiency. Battery SoC was taken as the state variable. Similarly, rule-based strategies were also considered in combination with ECMS, where optimization is carried out offline with DP in [35] and with more sophisticated multi-objective techniques, such as NSGA II in [25].

Optimization in HEVs often involves multi-objectives [14], and for a multi-objective optimization problem where the objectives are conflicting, multi-objective genetic algorithms are considered suitable [25], as they search for a Pareto optimal set. In [25], the two conflicting objectives of fuel consumption minimization and sizing were considered. When the configuration was evaluated, the parameters of the control strategy were optimized, whereas while evaluating the control strategy, it was manually tuned for minimum fuel consumption for one combination of drivetrain components. In [4], on the other hand, the best hybrid vehicle configuration and control strategy to reduce fuel consumption were identified at first. The control strategy was comprised of a set of rules based on both required power and battery and supercapacitor SoCs. Optimization goals included finding parameters that minimized fuel consumption and sustained battery SoC at the same time. The optimization results gave rise to a group of design parameters, indicating a compromise between fuel consumption and battery SoC.

Hybrid vehicles are often equipped with batteries as secondary storage elements. The battery aging process and its integration as a power management objective has already been discussed in [36]. The following material and text is based on [36]. In [37], an optimal control problem is formulated to minimize fuel consumption, as well as battery aging. With regards to battery lifetime, State of Health (SoH) estimation, commonly related to the battery capacity fade [38], is important. The maximum peak of the battery current is one of the factors influencing $\mathrm{SoH}$ and has to be limited. The number of battery recharge cycles during an interval have to be decreased as detailed in [39], but as mentioned in [39], this leads to more power generation from the primary source and, simultaneously, to higher fuel consumption. Thus, an inclusion of a second objective function as an optimization goal is necessary for extending the system's longevity without disregarding functionality. The two goals, fuel efficiency and battery aging, are conflicting objectives, as stated in [26]. Here, the requirement of high SoC for the minimization of total energy cost and the requirement of limited charging/discharging of the battery are the two conflicting objectives. In [26], two such conflicting objectives are evaluated for a plug-in hybrid electric vehicle charge pattern optimization. The optimal parameters corresponding to the objectives can be generated by optimization algorithms suitable for dealing with more than one objective.

In [26], these objectives are traded off using NSGA II. Here, the time, the maximum amount and the rate at which the HEV charges before each trip are taken as optimization variables. The consideration of both SoC and SoH is considered in [40], where, to carry out the task of optimal power management, 
a supervisor is presented. In [41,42], a genetic algorithm (GA) was used to fine-tune the parameters of a fuzzy logic controller. Three aspects of battery management were considered: minimization of resistive losses, yet supplying peak power demands, balanced battery energy in the entire duty cycle and controlling of the depth of charge/discharge for preserving the battery life.

In the field of hybrid electric vehicles (HEVs), power management and optimization strategies are not just restricted to those cases where the entire driving pattern is considered. In [18,21,43], for example, the optimal division of output torque is based on a specific driving pattern, and in [44], DP is considered for the optimization of several respective driving patterns. On the other hand, in [45], a prediction of the future driving pattern is considered based on past data. Use of human-in-the-loop in order to analyze driver requirements and the corresponding dynamic response of the vehicle is also not uncommon [46]. In [39], a fuzzy rule-based control was considered along with the prediction of the future path of the vehicle. Use of GPS for the knowledge of obstacles to come and the assumption of drive cycle being provided as a reference were one of the key aspects here. Apart from GPS, track-based prediction [47] and dynamic recurrent neural networks (DRNN) [48] have also been applied. Without the information available from telemetry, prediction strategies based on neural networks and stochastic Markov chain have been considered in [49] within an MPC framework. Past trajectories have been used for prediction, as considered in [48,50,51]. Here, a prediction algorithm considers certain features of the past trajectory measured over predefined time horizons. The adaption of horizon length (the number of past measurements) depending on the prediction performance is a key aspect here. The task of control optimization in [50] is carried out using MPC, and the results are compared to globally-optimal solutions determined by dynamic programming. In [52], an intelligent control, both with and without knowledge of the unknown, is considered; whereas in [53], control based on both the driving cycle, as well as driving style is elaborated. Within driving cycle-based control, methods to recognize present and to predict future driving conditions are detailed followed by methods for data analyses. In [54], an intelligent strategy based on the prediction of both the driving environment, as well as driving trends is considered. It consists of three major neural network systems: the first one for predicting road type and traffic congestion; the second one for predicting driving trend; the third one as a suite of intelligent networks, trained for all roadway types. In driving style-based control, methods to recognize drivers' driving styles, such as mild driving style, normal driving style, aggressive driving style, etc., are mentioned. The use of a driving simulator as an experimental platform is stated. In [55], a driving simulator has been used for improving driving efficiency and safety.

\section{Rule-Based Power Management and Optimization}

According to [33], the available power management optimization strategies can be categorized into three broad classes, namely global, static real time and dynamic real time. The rule-based control strategy is a basic control strategy that involves several modes of operation [33]. As detailed in [33], four operation modes are considered, namely motor and engine-only modes, hybrid braking mode and hybrid propelling mode. In these modes, an average constant charge is maintained in the storage component. Hence, SoC of the storage component is a constraint or determining factor for the switching between modes. Another factor is the desired torque. Three methods to improve rule-based control 
are discussed. The goal to be achieved is to optimize the vehicle's efficiency. In the first method, the modes are individually optimized on the basis of three criteria: optimal internal combustion engine (ICE) operation point, when to switch to electric-only mode and SoC maintenance, done by modifying/adding rules based on the three criteria. However, in [33], this is considered as not a very effective optimization method. The next optimization method for rule-based strategies is discussed: parameter optimization using genetic algorithms is termed as a static method suitable for determining globally-optimal values of a set of fixed parameters, such as upper and lower SoC bounds, etc. However, the inability of this optimization to dynamically control the parameters leads to the consideration of a third improvement method in [33], namely ECMS. Here, the storage efficiency or fuel to electric energy and the electric to motor efficiencies are determined in order to calculate the future savings in terms of the 'cost' of energy. An important advantage of combining with the ECMS approach is its real-time applicability in combination with prediction algorithms [30]. Finally, a real-time route-based optimization strategy is developed, capable of dynamically controlling the upper and lower bounds of SoC and the desired torque from the primary source based on pre-calculated optimal set points for the present and future. In [16], the controller not only has to assure the vehicles's power requirements, but also to minimize the energy consumption while maximizing the vehicle's autonomy. Here, offline calculation of optimal parameters for a rule-based controller is considered. Three machines, namely an electric motor, a generator and a battery, are considered, and modes are defined for a control strategy that will be able to manage the three different machines involved. Each mode has its own optimization problem definition, and the corresponding parameters can be offline optimized. This is detailed in Figure 1. Mode 1 is a full-electric mode, where the torque provided by the motor comes from the battery, and therefore, there are no degrees of freedom. Mode 2 is also an electric mode, but here, the generator assists the motor to propel the vehicle. This mode has one degree of freedom, and hence, the optimization problem concerns the efficiency maximization of both machines. Mode 3 is a range-extended mode, and here, both the engine and generator are used to produce power. The related optimization problem has two objectives: first, maximal production of electric energy by the generator and, second, minimal fuel consumption. Mode 4 is the power split mode, where all three machines are connected. As it is a combination of Modes 2 and 3, the optimal values calculated in the modes before can be used. The function of the controller is to select the most appropriate mode. The task of selecting modes is divided into first and second decision layers. In the first decision layer, SoC is the determining variable that decides whether to avoid battery use completely (safety reasons) or to switch between Modes 1, 2 (charge depleting) and 3, 4 (charge sustaining). The selection between Modes 1, 2, 3 and 4 is carried out in the second decision layer that incorporates the offline optimized values of the parameters, calculated for each mode. In [25], the rule-based controller is based on five operating modes. The driver torque request, speed of the crankshaft and SoC determine which mode is active. The optimization problem is divided into two sub-problems: optimization of the control strategy with the objective being fuel consumption minimization and optimization of component sizing. According to [25], only with the help of an appropriate control strategy, the fuel economy can be improved. This control strategy has to be adapted when the drivetrain configuration is evaluated. The evaluation of the configuration is done based on the hybridization factor (HF), which, in turn, affects the fuel economy and dynamic performance of the vehicle. This inter-dependence between goals is formulated in [25] as a multi-objective optimization 
problem. The solution is obtained in two steps: first, the optimization of the control strategy is carried out for different HF with fuel consumption minimization as the objective, leading to a set of parameters that are suitable for different HF; second, all of the following optimizations are carried out with a combined measure for fuel efficiency. In the optimization results, three kinds of solutions are marked: a good compromise of fuel consumption and performance, a bad compromise where the hybridization factor and fuel consumption are slightly lower, but with an increased acceleration time, and the best fuel consumption region, but with the worst acceleration.

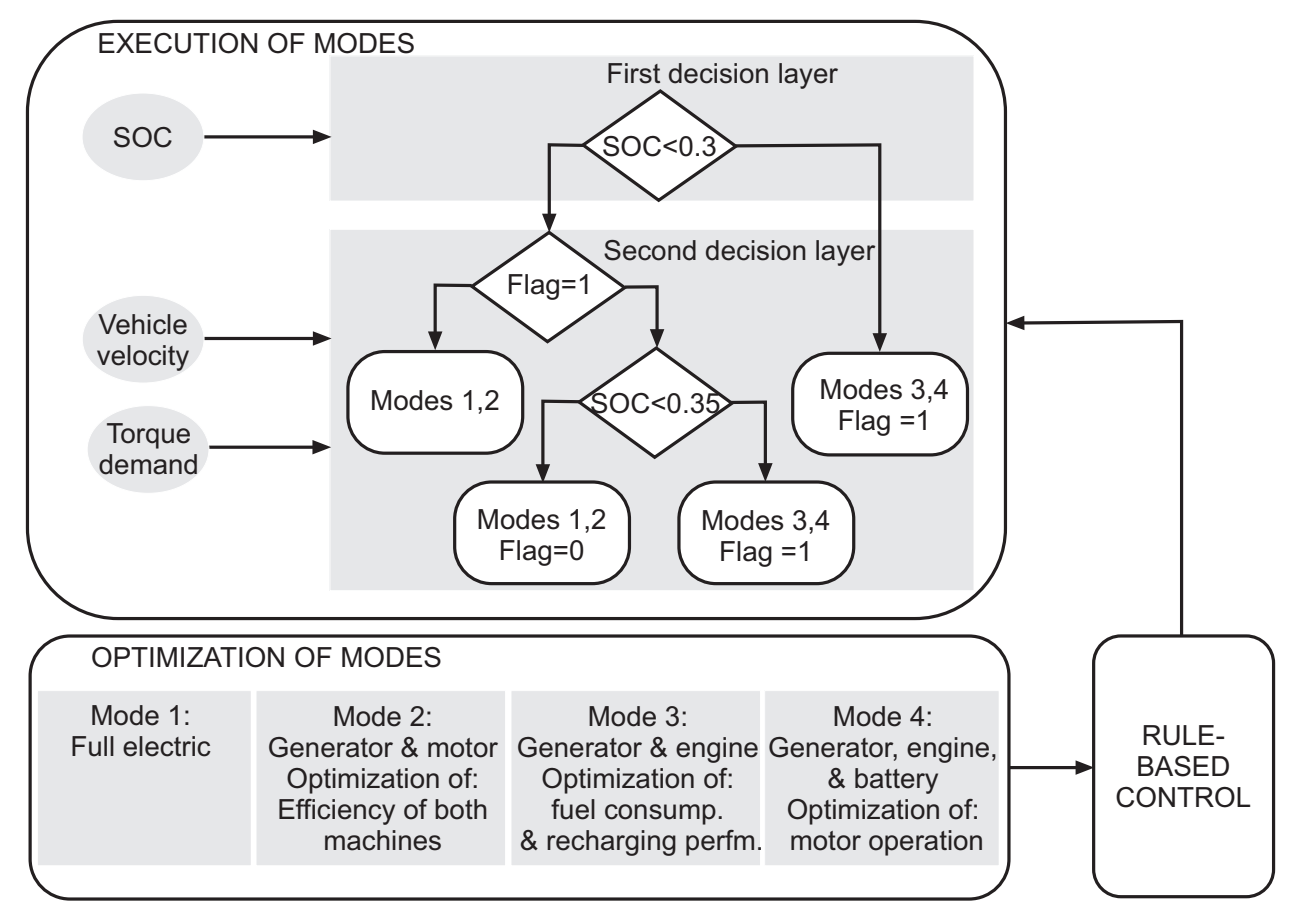

Figure 1. Rule-based controller for mode selection (according to [16]).

\section{Power Management Optimization with Battery Lifetime Management}

This section discusses topics that have already been elaborated in [36] and repeats the material. In terms of power management and optimization, fuel consumption minimization is the commonly known and accepted design goal. Rule-based power management often takes the battery SoC into account while designing the rules. In [56], based on battery $\mathrm{SoC}$ and $\mathrm{SoH}$, as well as other relevant parameters, the battery management system is able to deduce the electrical management. The results are communicated via controller area network (CAN bus) to the vehicle power management, which determines the power split between components while keeping the currents and voltages within the limits to safeguard the battery. According to [56,57], overcharging, over-discharging, mechanical stress and high temperature are some of the factors that lead to safety issues. The consideration of both SoC and $\mathrm{SoH}$ is also considered in [40]. The battery aging and degradation is limited by using SoC and $\mathrm{SoH}$ as constraints. The proposed supervisor consists of forecasting, predictive optimization and the local command stage. The forecast of inputs is sent from the forecasting stage to the optimization stage, which gives current commands to the DC/DC converter connected to the batteries at the local command stage. The physical parameters interacting at the local command stage are battery current and battery 
SoC and SoH. The battery model considered estimates SoC, taking into account charge variation as a function of current and temperature, $\mathrm{SoH}$ as a representation of capacity losses according to the battery depth of discharge and voltage as a function of SoC. The power management and optimization developed in [40] considers battery aging in the optimization process for a grid-connected PV system with batteries. The results show the reduction in grid power fluctuations, so that it is balanced to the power exchanged with the batteries.

At first, a non-optimal rule-based algorithm was developed taking into account the above-mentioned constraints. According to [40], the results obtained can be used as a reference for dynamic programming (DP). In [58], a fuzzy logic management system was tested in a real-time test-bench. A new quantity, called the battery working state (BWS), based on both battery terminal voltage and SoC, was used to make a decision on the power split. The control strategies were classified into rule-based and optimization-based. The advantages and disadvantages of both were stated, and the need to optimize rule-based strategies was emphasized. In [26], the conflicting objectives of fuel efficiency and battery aging are solved using a multi-objective solver, as shown in Figure 2. During charging, constant current and constant voltage cycles are applied on the battery model based on the optimized variables: time, maximum amount and the rate at which the HEV charges before each trip. During discharging, first, the drive cycle is given as the input to the vehicle model with available battery charge. Next, the current absorbed by the powertrain during the trip is recorded and sent to the electrochemical battery model. According to [26], this can help in evaluating the battery degradation while driving. During standstill conditions, battery degradation is calculated from the battery model alone. The two objectives are calculated at the end of the drive cycle and sent back to the optimizer, as shown in Figure 2.

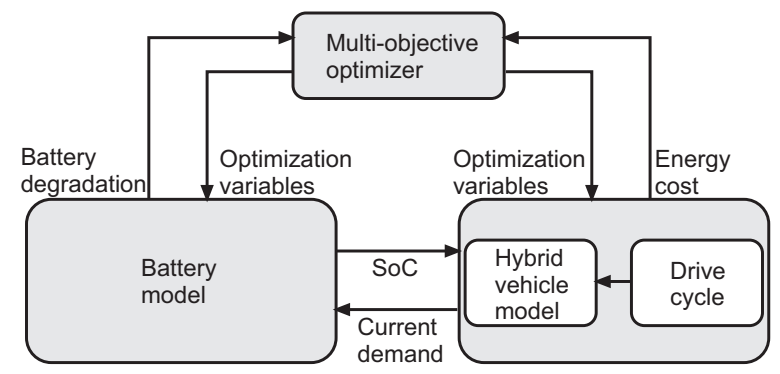

Figure 2. Optimization of battery charge patterns (according to [26]).

\section{Power Management Optimization with Unknown Velocity Input}

Apart from battery lifetime management, another issue with hybrid vehicles is the real-time applicability of power management optimization strategies. Under real driving conditions, no pre-defined drive cycle is followed, and optimal future driving behavior needs to be predicted. In [39], both predictive and protective algorithms are considered, where in the first part, the energy management based on the predicted future has fuel economy, performance and emission minimization as objectives; and in the second part, the modified energy management has battery $\mathrm{SoH}$ improvement as an additional objective. The controller based on fuzzy rules has two inputs, that is the differences between future and present speeds and positions. It has one output that manipulates the charge and discharge of the battery. Here, GPS has been used to acquire knowledge 
of the obstacles in the future, but in the absence of GPS, use of human-in-the-loop to analyze driver requirements and corresponding dynamic responses of the vehicle is also not uncommon [46]. In [46], a hardware test-bench used for HiLsimulations of HEV powertrain was coupled with an advanced virtual driving simulator. First, a forward facing model was selected. This kind of model requires controllers and a driver who can either track a given drive cycle or drive over a virtual scenario. The aim of using this model is to calculate vehicle speed and dynamic variables, such as forces in a forward direction along the powertrain. A real driver is asked to drive over a virtual scenario of 3 $\mathrm{km}$, and some dynamic variables were saved in order to analyze and re-design the powertrain control system later. Next, the backward facing model is tested. Here, the driving cycle information is also integrated, while the driver is driving over the same virtual scenario. Driving over the same driving scenario can be compared to a real case of a vehicle driven over the same commuting route every day, as given in [45]. According to [45], the future driving pattern can then be predicted based on past databases and a pattern matching system, where the database is used for storing past data and the pattern matching system for comparing current and past driving patterns and predicting the most likely future driving pattern. Since the driving patterns over a specific route are not unique, in [45], with the help of a clustering method, dividing driving patterns into certain classes is considered. Clustering is also considered in [53], where the task of collecting historical and current driving cycle data in order to analyze the previous driving pattern and to predict future driving conditions is elaborated. The importance of selecting a suitable length of time window is also stated. The methods for data analysis are detailed in [53]. In [43], an adaptive power management based on driving pattern recognition is presented. The driving pattern recognition algorithm classifies the given representative drive cycles (RDPs) based on low, medium and high power demands and creates driving patterns satisfying the characteristic parameters obtained from the driver velocity. The procedure followed is capable of executing an optimal online power management, along with driver velocity classification and prediction without using complicated algorithms stated in [53]. Here, two separate offline processes are considered: first is the RDP, where representative drive cycles are used to create six driving patterns satisfying certain criteria. These patterns are classified according to power demand and stored in a look-up table for online implementation; second is the driving pattern recognition (DPR), where the same six representative drive cycles are analyzed for determining the optimal power split that minimizes fuel consumption. Based on this, six control rules are formulated for a sub-optimal rule-based controller. This controller can now be implemented online. In the online process, the driver velocity is saved as historical data, and prediction is based on the assumption that the driving condition within a finite history will continue in the near future. The data in the most recent time frame from the historical data buffer can be used for characteristic parameter extraction. The classification of the driver velocity into six patterns is carried out based on the look-up table values from the first offline process (RDP). The corresponding control rule can also be determined based on the data from the second offline process (DPR). As shown in Figure 3, vehicle input signals are measured and control actions generated with sampling time "T". The duration of historical data sent from the buffer to the DPR process is "pT"; the duration of the RDP process is "fT"; and the duration of the control horizon is "NT". 


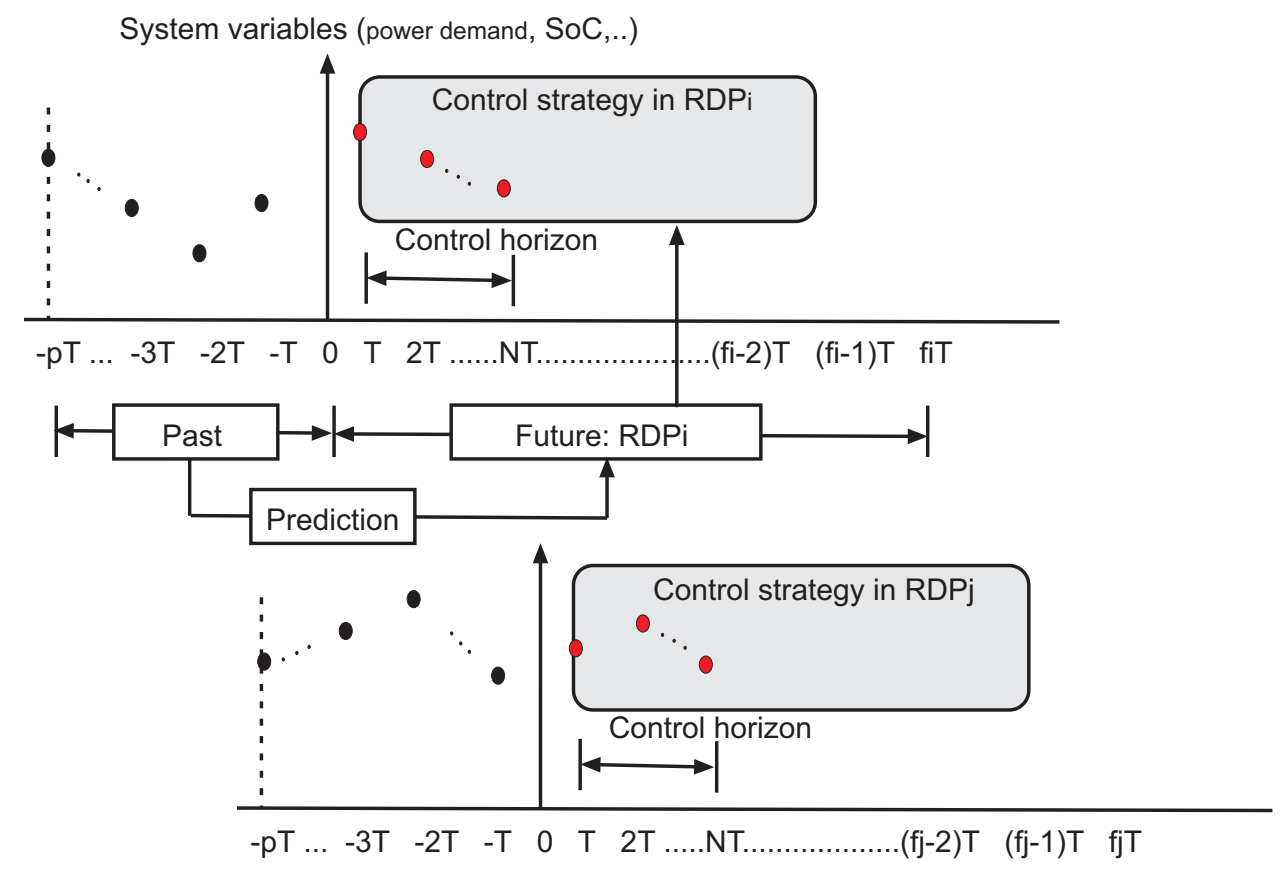

Figure 3. Driving pattern recognition and control (according to [43]).

\section{Need for a Generalized Optimal Rule-Based Control}

From the review of the published results, it can be stated that rule-based power management strategies are implementable in online, real-time control of hybrid electric vehicles. Their shortcomings in terms of non-optimality can be rectified by combining with global optimization methods, such as DP, GA, etc., to optimize various objectives, such as fuel consumption, etc. Rule-based strategies can also be combined with prediction algorithms and real-time control methods, such as ECMS, to provide route-based optimal control. Rule-based strategies often have rules formulated based on battery state-of-charge values, but since the rules are based on heuristics, they can also be modified to include state-of-health aspects with respect to the control of the aging of batteries. Maximum battery current being one of the factors affecting the state of health can lead to the development of a power management controller that can set limits to the rate of current increase, thereby minimizing the aging effects. The possibilities of individually optimizing the power management rules offline have already been considered in the literature. The results from optimization, stored in look-up tables, can be used to tune controller parameters online. Thus, depending on the particular optimization objective considered-minimization of fuel consumption, finding optimal current limits to minimize battery aging, or reducing the state-of-charge deviation, or a combination of these objectives - a suitable optimization can be carried out. Offline optimization with respect to these objectives makes the use of multi-objective optimization techniques plausible. Due to the flexibility of this rule-based controller, its application to a multi-source hybrid powertrain can be considered. For this, rules can be modified to include state-of-charge values of all of the storage components present. According to the literature, amongst multi-source hybrids, the use of a battery-supercapacitor combination can prove particularly beneficial. The main purpose of this literature research was to evaluate the potentials and application prospects of rule-based power management and 
to lay the foundations for the development of an appropriate optimal power management controller that takes into account multiple optimization objectives, in the presence of multiple sources.

\section{Conclusions}

In this contribution, an elaborate view of rule-based power management, its alternatives, optimization potentials and applications are given. First, an overview of HEVs and the advantages of selecting a suitable combination of components is given, followed by the details of optimizing rule-based power management. Then, optimization in the presence of multiple, complex and conflicting goals is discussed. Finally, the extent of rule-based power management in solving the issues of battery aging and dealing with real driving scenarios is reported with the help of relevant literature.

\section{Author Contributions}

Both authors contributed equally to the paper, whereby the corresponding author was mainly responsible for the initial writing, figures and literature research and the second author for structuring, organizing, reviewing and summarizing the entire contribution.

\section{Conflicts of Interest}

The authors declare no conflict of interest.

\section{References}

1. Khaligh, A.; Li, Z. Battery, ultracapacitor, fuel cell, and hybrid energy storage systems for electric, hybrid electric, fuel cell, and plug-in hybrid electric vehicles: State of the art. IEEE Trans. Veh. Technol. 2010, 59, 2806-2814 .

2. Bayindir, K.; Gozukucuk, M.A.; Teke, A. A comprehensive overview of hybrid electric vehicle: Powertrain configurations, powertrain control techniques and electronic control units. Energy Convers. Manag. 2010, 52, 1305-1313.

3. Aharon, I.; Kuperman, A. Topological overview of powertrains for battery-powered vehicles with range extenders. IEEE Trans. Power Electron. 2012, 26, 868-876.

4. Paladini, V.; Donateo, T.; Risi, A.; Laforgia, D. Super-capacitors fuel-cell hybrid electric vehicle optimization and control strategy development. Energy Convers. Manag. 2007, 48, 3001-3008.

5. Khoucha, F.; Benbouzid, M.E.H.; Kheloui, A. An optimal fuzzy logic power sharing strategy for parallel hybrid electric vehicles. In Proceedings of the IEEE Vehicle Power and Propulsion Conference (VPPC), Lille, France, 1-3 September 2010.

6. Li, Q.; Chen, W.; Li, Y.; Liu, S.; Huang, J. Energy management strategy for fuel cell/battery/ultracapacitor hybrid vehicle based on fuzzy logic. J. Electr. Power Energy Syst. 2012, 43, 514-525.

7. Kim, M.-J.; Peng, H. Power management and design optimization of fuel cell/battery hybrid vehicles. J. Power Sources 2007, 165, 819-832. 
8. Na, W.; Gou, B.; Kim, T. Analysis and control of a bidirectional DC/DC converter for an ultra-capacitor in a fuel cell generation system. J. Electr. Eng. Theory Appl. 2010, 1, 72-78.

9. Cao, J.; Emadi, A. A new battery/ultracapacitor hybrid energy storage system for electric, hybrid, and plug-in hybrid electric vehicles. IEEE Trans. Power Electr. 2012, 27, 122-132.

10. Gao, F.; Blunier, B.; Simoes, M.; Miraoui, A.; El-Moudni, A. PEM fuel cell stack hardware-in-the-loop emulation using DC/DC converter design. In Proceedings of the Electrical Power and Energy Conference (EPEC), Montreal, QC, Canada, 22-23 October 2009.

11. Özbek, M.; Wang, S.; Marx, M.; Söffker, D. Modeling and control of a PEM fuel cell system: A practical study based on experimental defined component behavior. J. Process Control 2013, 23, 282-293.

12. Moulik, B.; Söffker, D. Combined voltage-current control of DC/DC converters for power management of a multi-power source hybrid with HIL tests. In Proceedings of the ASME Dynamic Systems and Control Conference, San Antonio, TX, USA, 22-24 October 2014.

13. Salmasi, F.R. Control strategies for hybrid electric vehicles: Evolution, classification, comparison, and future trends. IEEE Trans. Veh. Technol. 2007, 56, 2393-2404.

14. Lin, C.; Filipi, Z.; Louca, L.; Peng, H.; Assanis, D.; Stein, J. Modelling and control of a medium-duty hybrid electric truck. Int. J. Veh. Des. 2004, 11, 349-370.

15. Mihael, C.; Pavković, D.; Petrić, J. A control-oriented simulation model of a power-split hybrid electric vehicle. Appl. Energy 2013, 101, 121-133.

16. Torres, J.L.; Gonzalez, R.; Gimenez, A.; Lopez, J. Energy management strategy for plug-in hybrid electric vehicles. A comparative study. Appl. Energy 2014, 114, 816-824.

17. Jalil, N.; Kheir, N.A.; Salman, M. A rule-based energy management strategy for a series hybrid vehicle. In Proceedings of the American Control Conference (ACC), Albuquerque, NM, USA, 4-6 June 1997.

18. Brahma, A.; Guezennec, Y.; Rizzoni, G. Optimal energy management in series hybrid electric vehicles. In Proceedings of the American Control Conference (ACC), Chicago, IL, USA, 28-30 June 2000.

19. Bin, Y.; Li, Y.; Gong, Q.; Peng, Z. Multi-information integrated trip specific optimal power management for plug-in hybrid electric vehicles. In Proceedings of the American Control Conference (ACC), St. Louis, MO, USA, 10-12 June 2009.

20. Pérez, L.V.; Bossio, G.R.; Moitre, D.; García, G.O. Optimization of power management in an hybrid electric vehicle using dynamic programming. Math. Comput. Simul. 2006, 73, 244-254.

21. Piccolo, A.; Ippolito, L.; Galdi, V.Z.; Vaccaro, A. Optimisation of energy flow management in hybrid electric vehicles via genetic algorithms. In Proceedings of the IEEE International Conference on Advanced Intelligent Mechatronics (IEEUASME), Como, Italy, 8-12 July 2001.

22. Jain, M.; Desai, C.; Williamson, S.S. Genetic algorithm based optimal powertrain component sizing and control strategy design for a fuel cell hybrid electric bus. In Proceedings of the IEEE Vehicle Power and Propulsion Conference (VPPC), Dearborn, MI, USA, 7-10 September 2009.

23. Deb, K.; Pratap, A.; Agarwal, S.; Meyarivan, T. A fast and elitist multi-objective genetic algorithm: NSGA-II. IEEE Trans. Evol. Comput. 2002, 6, 182-197. 
24. Zhang, B.; Chen, Z.; Mi, C.; Murphey, Y.L. Multi-objective parameter optimization of a series hybrid electric vehicle using evolutionary algorithms. In Proceedings of the IEEE Vehicle Power and Propulsion Conference (VPPC), Dearborn, MI, USA, 7-10 September 2009.

25. Buerger, S.; Lohmann, B.; Merz, M.; Vogel-Heuser, B.; Hallmannsegger, M. Multi-objective optimization of hybrid electric vehicles considering fuel consumption and dynamic performance. In Proceedings of the IEEE Vehicle Power and Propulsion Conference (VPPC), Lille, France, 1-3 September 2010.

26. Bashash, S.; Moura, S.J.; Forman, J.C.; Fathy, H.K. Plug-in hybrid electric vehicle charge pattern optimization for energy cost and battery longevity. J. Power Sources 2011, 196, 541-549.

27. Pagerit, S.; Rousseau, A.; Sharer, P. Global optimization to real time control of HEV power flow: Example of a fuel cell hybrid vehicle. In Proceedings of the 20th International Electric Vehicle Symposium (EVS20), Long Beach, CA, USA, 17-20 November 2005.

28. Kim, T.S.; Manzie, C.; Sharma, R. Model predictive control of velocity and torque split in a parallel hybrid vehicle. In Proceedings of the 2009 IEEE International Conference on Systems, Man, and Cybernetics, San Antonio, TX, USA, 11-14 October 2009.

29. Paganelli, G.; Delprat, S.; Guerra, T.M.; Rimaux, J.; Santin, J.J. Equivalent consumption minimization strategy for parallel hybrid powertrains. In Proceedings of the 55th IEEE Vehicular Technology Conference (VTS), Birmingham, AL, USA, 6-9 May 2002.

30. Guzzella, L.; Sciarretta, A. Vehicle Propulsion Systems: Introduction to Modeling and Optimization; Springer-Verlag: Berlin/Heidelberg, Germany, 2007.

31. Karbaschian, M.A.; Söffker, D. Review and comparison of power management approaches for hybrid vehicles with focus on hydraulic drives. Energies 2014, 7, 3512-3536.

32. Sciarretta, A.; Serrao, L.; Dewangan, P.C.; Tona, P.; Bergshoeff, E.N.D.; Bordons, C.; Charmpa, L.; Elbert, Ph.; Eriksson, L.; Hofman, T.; et al. A control benchmark on the energy management of a plug-in hybrid electric vehicle. Control Eng. Pract. 2014, 29, 287-298.

33. Meintz, A.; Ferdowsi, M. Control strategy optimization for a parallel hybrid electric vehicle. In Proceedings of the IEEE Vehicle Power and Propulsion Conference (VPPC), Harbin, China, 3-5 September 2008.

34. Sorrentino, M.; Rizzo, G.; Arsie, I. Analysis of a rule-based control strategy for on-board energy management of series hybrid vehicles. Control Eng. Pract. 2011, 19, 1433-1441.

35. Hofman, T.; Steinbuch, M. Rule-based energy management strategies for hybrid vehicles. Int. J. Electr. Hybrid Veh. 2007, 1, 71-94.

36. Beganovic, N.; Moulik, B.; Söffker, D. Li-O battery aging process: A smart review with respect to the integration of aging into system's power management. In Proceedings of the ASME Dynamic Systems and Control (DSC) Conference, Columbus, OH, USA, 28-30 October 2015.

37. Serrao, L.; Onori, S.; Sciarretta, A.; Guezennec, Y.; Rizzoni, G. Optimal energy management of hybrid electric vehicles including battery aging. In Proceedings of the American Control Conference (ACC), San Francisco, CA, USA, 29 June-1 July 2011.

38. Eddahech, A.; Briat, O.; Vinassa, J.M. Determination of lithium-ion battery state-of-health based on constant-voltage charge phase. J. Power Sources 2014, 258, 218-227. 
39. Hajimiri, M.H.; Salmasi, F.R. A fuzzy energy management strategy for series hybrid electric vehicle with predictive control and durability extension of the battery. In Proceedings of the IEEE Conference on Electric and Hybrid Vehicles (ICEHV), Pune, India, 18-20 December 2006.

40. Riffonneau, Y.; Bacha, S.; Barruel, F.; Ploix, S. Optimal power flow management for grid connected PV systems with batteries. IEEE Trans. Sustain. Energy 2011, 2, 309-320.

41. Wang, A.; Yang, W. Design of energy management strategy in hybrid vehicles by evolutionary fuzzy system part i: Fuzzy logic controller development. In Proceedings of the Sixth World Congress on Intelligent Control and Automation (WCICA), Dalian, China, 21-23 June 2006; Volume 2, pp. 8324-8328.

42. Wang, A.; Yang, W. Design of energy management strategy in hybrid vehicles by evolutionary fuzzy system part ii: Tuning fuzzy controller by genetic algorithms. In Proceedings of the Sixth World Congress on Intelligent Control and Automation (WCICA), Dalian, China, 21-23 June 2006; Volume 2, pp. 8329-8333.

43. Lin, C.C.; Jeon, S.; Peng, H.; Moo Lee, J. Driving pattern recognition for control of hybrid electric trucks. Veh. Syst. Dyn. 2004, 42, 41-58.

44. Park, Y.I.; Lee, J. Multi-mode driving control of a parallel hybrid electric vehicle using driving pattern recognition. J. Dyn. Syst. Meas. Control 2000, 124, 141-149.

45. Ichikawa, S.; Yokoi, Y.; Doki, S.; Okuma, S.; Naitou, T.; Shiimado, T.; Miki, N. Novel energy management system for hybrid electric vehicles utilizing car navigation over a commuting route. In Proceedings of the 2004 IEEE Intelligent Vehicles Symposium, Piscataway, NJ, USA, 14-17 June 2004; Volume 124, pp. 161-166.

46. Valera, J.J.; Iglesias, I.; Peña, A.; Martin, A.; Sánchez, J. Integrated modeling approach for highly electrified HEV. Virtual design and simulation methodology for advanced powertrain prototyping. In Proceedings of International Battery, Hybrid and Fuel Cell Electric Vehicle Symposium (EVS 24), Stavanger, Norway, 13-16 May 2009.

47. Cassebaum, O.; Biiker, B. Predictive supervisory control strategy for parallel HEVs using former velocity trajectories. In Proceedings of the IEEE Vehicle Power and Propulsion Conference (VPPC), Chicago, IL, USA, 6-9 September 2011.

48. Marx, M.; Shen, X.; Söffker, D. A data-driven online identification and control optimization approach applied to a hybrid electric powertrain system. IFAC Math. Model 2012, 7, 153-158.

49. Sun, C.; Hu, X.; Moura, S.J.; Sun, F. Velocity predictors for predictive energy management in hybrid electric vehicles. IEEE Trans. Control Syst. Technol. 2015, 23, 1197-1204.

50. Marx, M.; Söffker, D. Optimization of the powerflow control of a hybrid electric powertrain including load profile prediction. In Proceedings of the IEEE Vehicle Power and Propulsion Conference (VPPC), Seoul, Korea, 9-12 October 2012.

51. Söffker, D.; Wang, J.; Schiffer, S.; Marx, M.; Fu, X. Know your options-Interfacing consequences and forecasted performance analysis: A concept for the novel type of information system KYO-ICPA. In Proceedings of the IFAC/IFIP/IFORS/IEA Symposium on Analysis, Design, and Evaluation of Human-Machine Systems, Las Vegas, NV, USA, 11-15 August 2013.

52. Murphey, Y.L. Intelligent Vehicle Power Management: An Overview; Springer-Verlag: Berlin/Heidelberg, Germany, 2008. 
53. Wang, R.; Lukic, S.M. Review of driving conditions prediction and driving style recognition based control algorithms for hybrid electric vehicles. In Proceedings of the IEEE Vehicle Power and Propulsion Conference (VPPC), Chicago, IL, USA, 6-9 September 2011.

54. Park, J.; Chen, Z.; Murphey, Y.L. Intelligent vehicle power management through neural learning. In Proceedings of the International Joint Conference on Neural Networks, Barcelona, Spain, 18-23 July 2010.

55. Wang, J.; Moulik, B.; Söffker, D. Towards interactive driver assistance system to realize a safe and an efficient driving. In Proceedings of the IEEE-Vehicular Power and Propulsion Conference (VPPC), Montreal, QC, Canada, 19-22 October 2015.

56. Conte, F.V. Battery and battery management for hybrid electric vehicles: A review. $e$ \& i Elektrotechnik und Informationstechnik 2006, 123, 424-431.

57. Kitoh, K.; Remoto, H. 100 Wh large size Li-ion batteries and safety tests. J. Power Sources 1999, 2, 887-890.

58. Li, S.G.; Sharkh, S.M.; Walsh, F.C.; Zhang, C.N. Energy and battery management of a plug-in series hybrid electric vehicle using fuzzy logic. IEEE Trans. Veh. Technol. 2011, 60, 3571-3585.

(C) 2015 by the authors; licensee MDPI, Basel, Switzerland. This article is an open access article distributed under the terms and conditions of the Creative Commons Attribution license (http://creativecommons.org/licenses/by/4.0/). 